\title{
A New Multiuser Detector with Low Decision Delay for Variable Processing Gain DS/CDMA Mobile Radio Systems *
}

\author{
Ju Ho Lee and Hyung-Myung Kim \\ Department of Electrical Engineering, Korea Advanced Institute of Science and Technology \\ 373-1 Kusong-Dong Yusong-Gu Taejon 305-701, KOREA \\ Phone: +82-42-869-5440, 3440, Fax: +82-42-869-3410 \\ E-mail: jhlee@panda.kaist.ac.kr, hmkim@panda.kaist.ac.kr
}

\begin{abstract}
In this paper, an efficient multiuser detection scheme with low decision delay is proposed for asynchronous variable processing gain (VPG) DS/CDMA systems in mobile radio channels without any restrictions on processing gains. An equivalent synchronous single bit rate DS/CDMA system is first formulated to break up the detection problem into the blocks of finite length called processing windows. A low delay multipath-combining decisionfeedback multiuser detector (LDMCDF) is then proposed based on the equivalent system model. Since the LDMCDF makes the decisions for data bits every processing window, the decision delay is less than the interval of one processing window. The effect of the processing window length on the performance of the LDMCDF is evaluated, and the simulation results show that the LDMCDF provides good performance with the negligible decision delay.
\end{abstract}

\section{INTRODUCTION}

The multiple access interference (MAI) and nearfar problem limit the capacity and performance of DS/CDMA systems significantly. There has been a great deal of interest in improving DS/CDMA detection through the use of multiuser detectors [1].

Future DS/CDMA systems must be able to support multi-rate services such as speech, image, data, and even moving picture. Therefore, receiver design for multi-rate DS/CDMA systems is a research area of great interest. In order to transmit signals with multiple data rates sharing a given bandwidth, the most easiest is the variable processing gain (VPG) system, which alters the processing gain according to bit rates and spread all signals to the same bandwidth [2]. There have been several studies [3]-[6] on receiver design for the VPG-DS/CDMA systems. In all of those studies, however, data rates are assumed to be the integer multiples of the lowest data rate. Furthermore, [3]-[5] assumed the synchronous system. The receiver proposed in [6], although the asynchronous system was considered, may result in large decision delay for the high data rate user if this scheme is applied to the system of the wide range of data rates.

In this paper, we consider the multiuser detection scheme for the asynchronous VPG-DS/CDMA system in the frequency-selective slowly-varying Rayleigh fading channel without any restrictions on the processing gain. A multiuser detector which

*The authors wish to acknowledge the financial support of the Korea Research Foundation made in the program year of 1998. achieves good performance with negligible decision delay is developed. In order to reduce the decision delay, we break up the detection problem into the finite length blocks called processing windows by deriving an equivalent synchronous single rate DS/CDMA system model. Based on this equivalent system model, a low delay multipath-combining decision-feedback multiuser detector (LDMCDF) is proposed. The multipath signals are coherently combined to alleviate the fading effects of the channel. The MAI is canceled by applying the partial decorrelating decision-feedback scheme [7] to the combined multipath signals. Thus, the complexity of the MAI cancellation does not depend on the number of paths being combined. The decisions of the transmitted data bits are obtained through the maximal-ratio combining every processing window. Hence, the decision delay becomes less than the interval of one processing window.

\section{Received Signal Model}

Consider the asynchronous VPG-DS/CDMA system with $K$ users. The bit duration of the $k$ th user is $T_{k}=L_{k} T_{c}$, where $L_{k}$ is the processing gain and $T_{c}$ is the chip duration. No special restrictions are made on $L_{k}$. The $j$ th bit transmitted by the $k$ th user is denoted by $b_{k, j}$. The signature waveform for the $j$ th bit of the $k$ th user $a_{k, j}(t)$, which is restricted to the bit interval of duration $T_{k}$, is assumed to be real and to satisfy $\int_{0}^{T_{k}}\left|a_{k, j}(t)\right|^{2} d t=1$. The signal received through the frequency-selective channel can be represented by using the tapped-delay-line model [8] as

$$
\begin{aligned}
r(t)= & n(t)+\sum_{k=1}^{K} \sum_{j=-\infty}^{\infty} \sum_{m=0}^{M-1} \lambda_{k, j}^{(m)} \sqrt{2 E_{k}} b_{k, j} \\
& a_{k, j}\left(t-j T_{k}-m T_{c}-\tau_{k}\right) \cos \left(\omega_{k} t+\phi_{k, j}^{(m)}\right),
\end{aligned}
$$

where $n(t)$ is the white Gaussian noise with two-sided power spectral density $N_{o} / 2$. In (1), $E_{k}, \omega_{k}$, and $\tau_{k}$ are the transmitted bit energy, carrier frequency, and relative time delay, respectively, of user $k$. Let $\Delta$ denote the multipath delay spread. Then, the number of resolvable paths, $M$, can be approximated as $M=$ $\left\lfloor\Delta / T_{c}\right\rfloor+1$, where $\lfloor x\rfloor$ denotes the largest integer less than or equal to $x$. The gain of the $m$ th path for the $j$ th bit of user $k, \lambda_{k, j}^{(m)}$, is assumed to have Rayleigh distribution. The received phase $\phi_{k, j}^{(m)}$ is assumed to 
be uniformly distributed over $[0,2 \pi)$. The received signal in (1) can be rewritten in terms of the quadratic components as

$$
\begin{aligned}
r(t)= & \sum_{k=1}^{K} \sum_{j=-\infty}^{\infty} b_{k, j} \sqrt{2 E_{k}}\left\{s_{k, j}^{I}\left(t-j T_{k}\right) \cos \left(\omega_{k} t\right)\right. \\
& \left.-s_{k, j}^{Q}\left(t-j T_{k}\right) \sin \left(\omega_{k} t\right)\right\}+n(t)
\end{aligned}
$$

where

$$
\begin{aligned}
& s_{k, j}^{I}(t)=\sum_{m=0}^{M-1} \lambda_{k, j}^{(m)} a_{k, j}\left(t-m T_{c}-\tau_{k}\right) \cos \left(\phi_{k, j}^{(m)}\right), \\
& s_{k, j}^{Q}(t)=\sum_{m=0}^{M-1} \lambda_{k, j}^{(m)} a_{k, j}\left(t-m T_{c}-\tau_{k}\right) \sin \left(\phi_{k, j}^{(m)}\right) .
\end{aligned}
$$

$s_{k, j}^{I}(t)$ and $s_{k, j}^{Q}(t)$ can be considered as the received signature waveforms for in-phase and quadrature phase component, respectively, of the signal passed through the multipath fading channel.

\section{Equivalent Synchronous Single Rate DS/CDMA SySTEM MOdEL}

Let the time interval $\left[i T_{w},(i+1) T_{w}\right)$ be called as the $i$ th processing window. The length of the processing window can be determined according to system requirements such as the decision delay and bit error rate (BER) performance.

Depending upon the bit rates, some bits are received during a single processing window and other bits during several processing windows. The maximum number of the processing windows with which a single bit of user $k$ can overlap is given by $G_{k}+1$, in which $G_{k}=\left\lceil\left(T_{k}+(M-1) T_{c}\right) / T_{w}\right\rceil$, where $\lceil x\rceil$ denotes the smallest integer greater than or equal to $x$. Let $G=\max \left\{G_{1}, G_{2}, \cdots, G_{K}\right\}$. Then, the reception of any bit beginning with the $i$ th processing window is completed before the $(i+G)$ th processing window ends.

Let a physical user denote the user in the original system and an effective user denote the imaginary user in the proposed equivalent system model. During the $i$ th processing window, each bit of physical users overlapped with the $i$ th processing window is mapped to one effective user according to the completion time of reception of it. If the reception of a physical user bit ends in the $(i+g)$ th processing window, then this bit is mapped to an effective user belonging to group $\boldsymbol{B}_{g}(i)$ (see Fig. 1), where $g=0,1, \cdots, G$. Therefore, each effective user transmits one data bit during the interval of one processing window, and the bit rate of the equivalent system is $1 / T_{w}$. It is noted that if a physical user bit overlaps with $N$ processing windows (see Fig. 1), then this bit generates $N$ effective users (one effective user in each processing window) which represent the same physical data.

The signature waveform for an effective user in the $i$ th processing window is the same as the received one for the corresponding physical user bit during the interval occupied by this bit (see the shaded region in Fig. 1) and is zero in the rest of the $i$ th processing window. The other parameters of effective users are also obtained from the parameters of the corresponding bits of physical users. In order to use the decisionfeedback detection scheme [7], the effective users of each group are reordered in such a way that the received energies of the corresponding bits of physical users might be in non-increasing order. The received signal in (2) can be rewritten in terms of effective users as

$$
\begin{aligned}
r(t)= & \sum_{i=-\infty}^{\infty} \sum_{g=0}^{G} \sum_{p=1}^{K_{g}(i)} b_{p}^{(g)}(i) \sqrt{2 E_{p}^{(g)}(i)}\left\{\tilde{s}_{p}^{I(g)}(i, t)\right. \\
& \left.\cos \left(\omega_{p}^{(g)}(i) t\right)-\tilde{s}_{p}^{Q(g)}(i, t) \sin \left(\omega_{p}^{(g)}(i) t\right)\right\} \\
& +n(t),
\end{aligned}
$$

where $K_{g}(i)$ denotes the number of effective users of $B_{g}(i) . \quad$ In $(3), b_{p}^{(g)}(i), \tilde{s}_{p}^{I(g)}(i, t)$, and $\tilde{s}_{p}^{Q(g)}(i, t)$ are the data bit, in-phase signature waveform, and quadrature phase signature waveform, respectively, of the $p$ th effective user of $\boldsymbol{B}_{g}(i)$. The carrier frequency, $\omega_{p}^{(g)}(i)$, and transmitted bit energy, $E_{p}^{(g)}(i)$, of the $p$ th effective user of $\boldsymbol{B}_{g}(i)$ are the carrier frequency and transmitted bit energy, respectively, of the corresponding physical user. As shown in (3), during the $i$ th processing window, the asynchronous VPG-DS/CDMA system with $K$ physical users can be represented as the equivalent synchronous single rate DS/CDMA system with $\tilde{K}(i)=\sum_{g=0}^{G} K_{g}(i)$ effective users.

In the rest of this section, the simple vector-matrix expression is derived for the proposed LDMCDF. For the notational simplicity, when $\tilde{K}(i)$ and $K_{g}(i)$ are used as a subscript or as a superscript, we will use $\tilde{K}$ and $K_{g}$ instead, respectively.

The fading effect can be compensated by employing multipath diversity combiners known as RAKE receivers in DS/CDMA systems [8]. The number of diversity branches of the RAKE receiver is assumed to be equal to that of resolvable paths. Then, with the perfect estimation of the channel parameters, the output of the RAKE receiver for the $p$ th effective user of $\boldsymbol{B}_{g}(i), y_{p}^{(g)}(i)$, is given by

$$
y_{p}^{(g)}(i)=y_{p}^{I(g)}(i)+y_{p}^{Q(g)}(i)
$$

where

$$
\begin{gathered}
y_{p}^{I(g)}(i)=\int_{i T_{w}}^{(i+1) T_{w}} r(t) \sqrt{2} \tilde{s}_{p}^{I(g)}(i, t) \cos \left(\omega_{p}^{(g)}(i) t\right) d t \\
y_{p}^{Q(g)}(i)=\int_{i T_{w}}^{(i+1) T_{w}}-r(t) \sqrt{2} \tilde{s}_{p}^{Q(g)}(i, t) \sin \left(\omega_{p}^{(g)}(i) t\right) d t .
\end{gathered}
$$

Let $\mathbf{b}^{(g)}(i)=\left[b_{1}^{(g)}(i), \cdots, b_{K_{g}}^{(g)}(i)\right]^{T}$ denote the data bit vector for the effective users of $\boldsymbol{B}_{g}(i), \mathbf{W}^{(g)}(i)=$ 


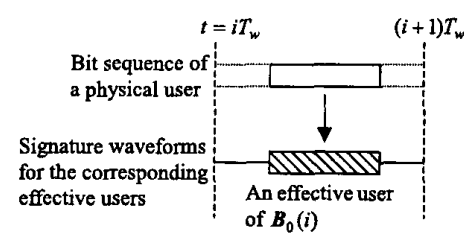

(a)

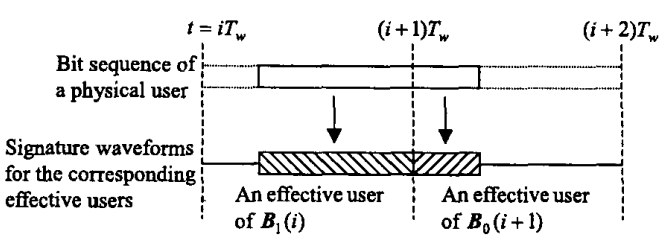

(b)

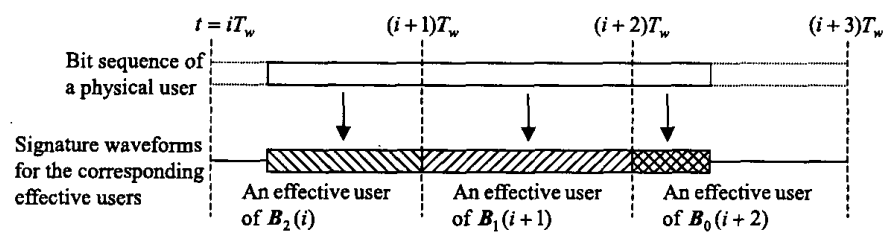

(c)

Fig. 1. Examples on the mapping between the physical user bit and the effective user. (a) A physical user bit overlapped with one processing window. (b) A physical user bit overlapped with two processing windows. (c) A physical user bit overlapped with three processing windows.

$\operatorname{diag}\left[\left(E_{1}^{(g)}(i)\right)^{1 / 2}, \cdots,\left(E_{K_{g}}^{(g)}(i)\right)^{1 / 2}\right]$ the transmitted bit energy matrix, and $\mathbf{y}^{(g)}(i)=\left[y_{1}^{(g)}(i), \cdots, y_{K_{g}}^{(g)}(i)\right]^{T}$ the sampled output vector of the RAKE receiver bank. Let $\mathbf{H}^{(g, h)}(i) \in \mathbb{R}^{K_{g} \times K_{h}}$ represent the crosscorrelation matrix of the signature waveforms for the effective users of $\boldsymbol{B}_{g}(i)$ and $\boldsymbol{B}_{h}(i)$ with its $(p, q)$ th element given by

$$
\begin{aligned}
& H_{p, q}^{(g, h)}(i)= \\
& \int_{i T_{w}}^{(i+1) T_{w}} \tilde{s}_{p}^{I(g)}(i, t) \tilde{s}_{q}^{I(h)}(i, t) \cos \left(\Delta \omega_{p, q}^{(g, h)}(i) t\right) \\
& +\tilde{s}_{p}^{I(g)}(i, t) \tilde{s}_{q}^{Q(h)}(i, t) \sin \left(\Delta \omega_{p, q}^{(g, h)}(i) t\right) \\
& -\tilde{s}_{p}^{Q(g)}(i, t) \tilde{s}_{q}^{I(h)}(i, t) \sin \left(\Delta \omega_{p, q}^{(g, h)}(i) t\right) \\
& +\tilde{s}_{p}^{Q(g)}(i, t) \tilde{s}_{q}^{Q(h)}(i, t) \cos \left(\Delta \omega_{p, q}^{(g, h)}(i) t\right) d t,
\end{aligned}
$$

where $\Delta \omega_{p, q}^{(g, h)}(i)=\omega_{p}^{(g)}(i)-\omega_{q}^{(h)}(i)$. Then, the overall sampled output vector, $\mathbf{y}(i)$, of the RAKE receiver bank for the effective users during the $i$ th processing window is given by

$$
\mathbf{y}(i)=\mathbf{H}(i) \mathbf{W}(i) \mathbf{b}(i)+\mathbf{n}(i),
$$

where $\mathbf{y}(i)=\left[\left(\mathbf{y}^{(0)}(i)\right)^{T}, \cdots,\left(\mathbf{y}^{(G)}(i)\right)^{T}\right]^{T}, \mathbf{b}(i)=$ $\left[\left(\mathbf{b}^{(0)}(i)\right)^{T}, \cdots,\left(\mathbf{b}^{(G)}(i)\right)^{T}\right]^{T}$, and

$$
\begin{aligned}
\mathbf{H}(i) & =\left[\begin{array}{lll}
\mathbf{H}^{(0,0)}(i) & \cdots & \mathbf{H}^{(0, G)}(i) \\
\vdots & \ddots & \vdots \\
\mathbf{H}^{(G, 0)}(i) & \cdots & \mathbf{H}^{(G, G)}(i)
\end{array}\right], \\
\mathbf{W}(i) & =\left[\begin{array}{lll}
\mathbf{W}^{(0)}(i) & \cdots & \mathbf{0}_{K_{0} \times K_{G}} \\
\vdots & \ddots & \vdots \\
\mathbf{0}_{K_{G} \times K_{0}} & \cdots & \mathbf{W}^{(G)}(i)
\end{array}\right],
\end{aligned}
$$

and $\mathbf{n}(i) \in \mathbb{R}^{\tilde{K} \times 1}$ is the zero-mean Gaussian noise vector with autocorrelation matrix $\mathbf{R}(\mathbf{n}(i))=\frac{N_{o}}{2} \mathbf{H}(i)$.

\section{Low Delay Multipath-Combining Decision-Feedback Multiuser Detector}

The receiver structure is presented in Fig. 2. We assume the perfect estimation of timing and channel parameters. During the $i$ th processing window, oneto-one mappings between the effective users and the bits of physical users are found first. Then, the parameters of the effective users are derived by using the one-to-one mappings and the estimated channel parameters. During the $i$ th processing window, the LDMCDF makes the decisions for the effective users of $\boldsymbol{B}_{0}(i)$, whose corresponding physical user bits are completely received before the $i$ th processing window ends, and the decisions for physical users are obtained through the inverse mapping. The LDMCDF removes the MAI by using the partial decorrelating decisionfeedback scheme [7]. The detailed description on the MAI cancellation is given in the rest of this section.

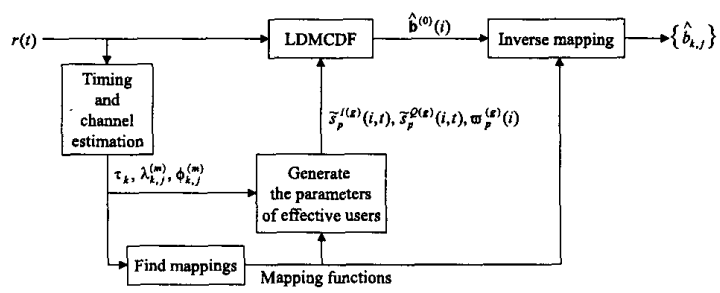

Fig. 2. Receiver structure.

$\mathbf{H}(i)$ in (5) is assumed to be a symmetric positive definite matrix. Then, $\mathbf{H}(i)$ can be factored as $\mathbf{H}(i)=\mathbf{F}^{T}(i) \mathbf{F}(i)$, where $\mathbf{F}(i)$ is a lower triangular matrix with positive diagonal elements (see Cholesky factorization in [9]). By applying a whitening filter $\left(\mathbf{F}^{T}(i)\right)^{-1}$ to $\mathbf{y}(i)$ in (5), the MAI due to the effective users of $\boldsymbol{B}_{1}(i), \cdots, \boldsymbol{B}_{G}(i)$, whose decisions can 
not be obtained during the $i$ th processing window, is removed from $\mathbf{y}^{(0)}(i)$. Since $\mathbf{F}(i)$ can be represented in terms of $\mathbf{F}^{(g, h)}(i) \in \mathbb{R}^{K_{g} \times K_{h}}$ as follows,

$$
\mathbf{F}(i)=\left[\begin{array}{lll}
\mathbf{F}^{(0,0)}(i) & \cdots & \mathbf{0}_{K_{0} \times K_{G}} \\
\vdots & \ddots & \vdots \\
\mathbf{F}^{(G, 0)}(i) & \cdots & \mathbf{F}^{(G, G)}(i)
\end{array}\right]
$$

the output of the whitening filter for the effective users of $\boldsymbol{B}_{g}(i), \tilde{\mathbf{y}}^{(g)}(i)$, can be expressed as

$$
\tilde{\mathbf{y}}^{(g)}(i)=\sum_{h=0}^{g} \mathbf{F}^{(g, h)}(i) \mathbf{W}^{(h)}(i) \mathbf{b}^{(h)}(i)+\tilde{\mathbf{n}}^{(g)}(i),
$$

where $\tilde{\mathbf{n}}^{(g)}(i) \in \mathbb{R}^{K_{g} \times 1}$ is the zero-mean Gaussian noise vector with autocorrelation matrix $\mathbf{R}\left(\tilde{\mathbf{n}}^{(g)}(i)\right)=$ $\frac{N_{o}}{2} \mathbf{I}_{K_{g}}$. We note that $\mathbf{F}(i)$ and, hence, $\mathbf{F}^{(g, g)}(i)$ are lower triangular matrices with positive diagonal elements.

Let $D_{p}(i)$ denote the number of the previous processing windows with which the physical user bit corresponding to the $p$ th effective user of $\boldsymbol{B}_{0}(i)$ overlaps, and $D(i)=\max \left\{D_{1}(i), \cdots, D_{K_{0}}(i)\right\}$. Then, there exist effective users which belong to $\boldsymbol{B}_{\boldsymbol{d}}(i-d)$ and represent the same physical data as the $p$ th effective user of $\boldsymbol{B}_{0}(i)$, for $d=1, \cdots, D_{p}(i) .{ }^{1}$ Define $p^{\prime}(d)$ such that the $p^{\prime}(d)$ th effective user of $\boldsymbol{B}_{d}(i-d)$ represents the same physical data as the $p$ th effective user of $\boldsymbol{B}_{0}(i)$, for $d=1, \cdots, D_{p}(i)$, and $p^{\prime}(0)=p$. For the notational simplicity, $p^{\prime}$ will be used instead of $p^{\prime}(d)$ when it is used as a subscript. For $d=0, \cdots, D_{p}(i)$, the whitening filter output, $\tilde{y}_{p^{\prime}}^{(d)}(i-d)$, for the $p^{\prime}(d)$ th effective user of $\boldsymbol{B}_{d}(i-d)$ contains the information on $b_{p^{\prime}}^{(d)}(i-d)$, or the information on $b_{p}^{(0)}(i)$. Therefore, in order to make the decision $\hat{b}_{p}^{(0)}(i)$ for $b_{p}^{(0)}(i)$, $\tilde{y}_{p^{\prime}}^{(d)}(i-d)$ for $d=0, \cdots, D_{p}(i)$ should be used jointly. Since the above statement holds for all effective users of $\boldsymbol{B}_{0}(i), \tilde{\mathbf{y}}^{(d)}(i-d), 0 \leq d \leq D(i)$, should be utilized jointly to obtain the decision $\hat{\mathbf{b}}^{(0)}(i)$ for $\mathbf{b}^{(0)}(i)$, where $\tilde{\mathbf{y}}^{(d)}(i-d)$ is given by

$$
\begin{aligned}
\tilde{\mathbf{y}}^{(d)}(i-d)= & \sum_{g=0}^{d} \mathbf{F}^{(d, g)}(i-d) \mathbf{W}^{(g)}(i-d) \\
& \mathbf{b}^{(g)}(i-d)+\tilde{\mathbf{n}}^{(d)}(i-d) .
\end{aligned}
$$

From (8), it can be seen that the MAI terms in $\tilde{y}_{p^{\prime}}^{(d)}(i-d)$ consist of the intergroup MAI due to the effective users of $\boldsymbol{B}_{0}(i-d), \cdots, \boldsymbol{B}_{d-1}(i-d)$ and the intragroup MAI due to the effective users $1, \cdots, p^{\prime}(d)-1$ of $\boldsymbol{B}_{d}(i-d)$.

Since $\hat{\mathbf{b}}^{(0)}(l)$ for $l<i$ have been obtained prior to the $i$ th processing window and $\hat{\mathbf{b}}^{\left(l^{\prime}\right)}\left(l-l^{\prime}\right)$ for $l^{\prime}=1, \cdots, D(l)$ can be obtained from $\hat{\mathbf{b}}^{(0)}(l)$, the

${ }^{1}$ Let $\mathcal{S}\left(\boldsymbol{B}_{g}(i)\right)$ denote the set of physical data represented by the effective users of $\boldsymbol{B}_{g}(i)$. Then, $\mathcal{S}\left(\boldsymbol{B}_{d}(i-d)\right)$ is the subset of $\mathcal{S}\left(\boldsymbol{B}_{d-1}(i-d+1)\right)$, for $d=1, \cdots, D(i)$. This fact implies that $\mathcal{S}\left(\boldsymbol{B}_{D(i)}(i-D(i))\right) \subset \mathcal{S}\left(\boldsymbol{B}_{D(i)-1}(i-D(i)+1)\right) \subset \cdots \subset$ $\mathcal{S}\left(\boldsymbol{B}_{0}(i)\right)$. decisions for $\mathbf{b}^{(0)}(i-d), \cdots, \mathbf{b}^{(d-1)}(i-d)$ are available during the $i$ th processing window. Therefore, the intergroup MAI can be removed from $\tilde{\mathbf{y}}^{(d)}(i-d)$ by using $\hat{\mathbf{b}}^{(0)}(i-d), \cdots, \hat{\mathbf{b}}^{(d-1)}(i-d)$. The output, $\mathbf{u}^{(d)}(i)$, obtained by canceling the intergroup MAI, is given by

$$
\begin{gathered}
\mathbf{u}^{(d)}(i)=\tilde{\mathbf{y}}^{(d)}(i-d)-\sum_{g=0}^{d-1} \mathbf{F}^{(d, g)}(i-d) \\
\mathbf{W}^{(g)}(i-d) \hat{\mathbf{b}}^{(g)}(i-d) .
\end{gathered}
$$

The decisions for the effective users of $\boldsymbol{B}_{0}(i)$ are obtained in the increasing order of the indices of effective users, i.e. from the 1st effective user to the $K_{0}(i)$ th one, by using the decision-feedback detection, based on $\mathbf{u}^{(d)}(i)$ for $d=0, \cdots, D(i)$. And, $\hat{b}_{p^{\prime}}^{(d)}(i-d)=\hat{b}_{p}^{(0)}(i)$ for $d=1, \cdots, D_{p}(i)$. In the decision-making process, therefore, the decisions for the effective users $1, \cdots, p^{\prime}(d)-1$ of $B_{d}(i-d)$ are obtained prior to that for the $p^{\prime}(d)$ th effective user of $\boldsymbol{B}_{d}(i-d)$. Hence, the intragroup MAI remaining in $u_{p^{\prime}}^{(d)}(i)$ can be canceled as follows,

$$
\begin{aligned}
z_{p}^{(d)}(i)= & u_{p^{\prime}}^{(d)}(i)-\sum_{q=1}^{p^{\prime}(d)-1} F_{p^{\prime}, q}^{(d, d)}(i-d) \\
& W_{q, q}^{(d)}(i-d) \hat{b}_{q}^{(d)}(i-d) .
\end{aligned}
$$

In the case that all feedback decisions are correct, $z_{p}^{(d)}(i)$ in (10) contains no MAI terms. Since $z_{p}^{(d)}(i)$ contains the information on $b_{p}^{(0)}(i), 0 \leq d \leq D_{p}(i)$, the decision for $b_{p}^{(0)}(i)$ is obtained by using the maximal-ratio combining of $z_{p}^{(d)}(i)$ to recover the received bit energy as

$$
\hat{b}_{p}^{(0)}(i)=\operatorname{sgn}\left(\sum_{d=0}^{D_{p}(i)} \alpha_{p}^{(d)}(i) z_{p}^{(d)}(i)\right),
$$

where the maximal-ratio combining coefficient $\alpha_{p}^{(d)}(i)$ is given by

$$
\alpha_{p}^{(d)}(i)=F_{p^{\prime}, p^{\prime}}^{(d, d)}(i-d) W_{p^{\prime}, p^{\prime}}^{(d)}(i-d) .
$$

\section{Simulation Results}

In the simulation, it is assumed that the chip waveform is of the rectangular pulse type and each bit uses an identical signature waveform. It is also assumed that the mean power of the path gain decreases by 1 [dB] as the path delay increases by one chip duration and the mean received bit energy, $E_{b}$, is the same for each user. The number of users for each bit rate is assumed to be the same. The performance of the LDMCDF is compared with those of the conventional RAKE receiver bank (CRAKEB), the conventional RAKE receiver in the single user system (CRAKESU), and the multipath-decorrelating maximal-ratio combining receiver (MD-MRC) [10]. 


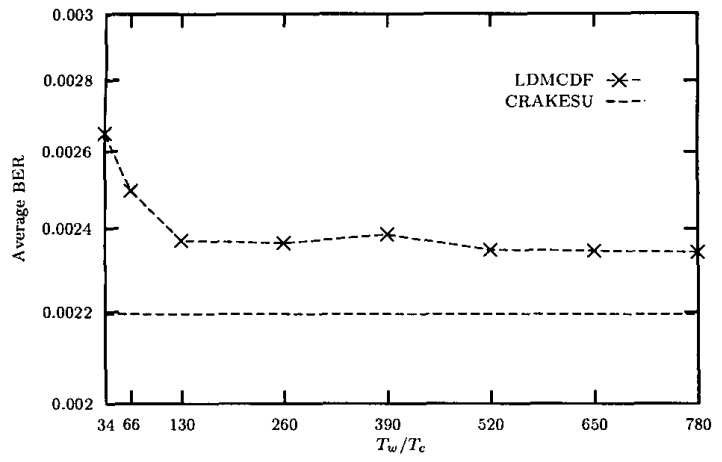

Fig. 3. Effect of the processing window length.

In Fig. 3, the average BER of the LDMCDF is plotted as the function of $T_{w} / T_{c}$ with $E_{b} / N_{o}=9$ [dB]. Gold sequences are used as the signature sequences. The processing gains are $L=31,63,127$, and the number of users is $K=12$. It is assumed that there are $M=4$ resolvable paths. Note that the first three values of $T_{w}\left(T_{w} / T_{c}=34,66,130\right)$ correspond to the time intervals during which a single bit of each bit rate is received. As the processing window length becomes long, the number of the effective users of $\boldsymbol{B}_{0}(i)$ increases, and so does the average number of interfering bits of which decisions are used as the feedback terms. Thus, as $T_{w}$ becomes large, the decisionfeedback gain increases, i.e., the BER performance of the LDMCDF improves, although the decision delay increases. When $T_{w}$ is greater than the maximum reception interval, the decisions of almost all the interfering bits are utilized as the feedback terms. Hence, the increase in $T_{w}$ results in little performance improvement.

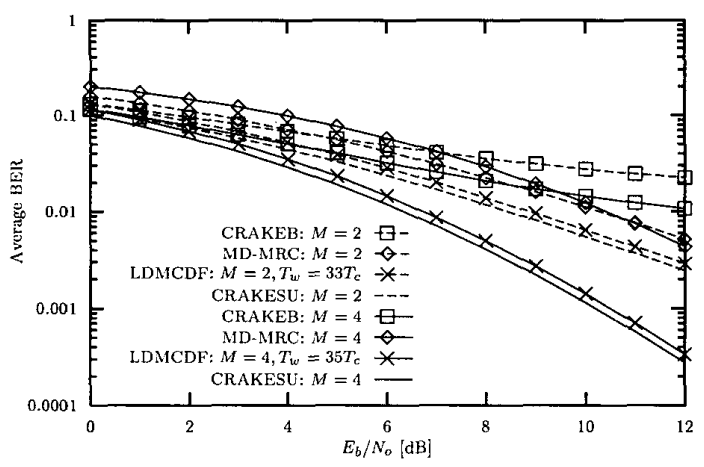

Fig. 4. Comparison between the average BERs of the LDMCDF and the MD-MRC.

Fig. 4 shows the average BERs against $E_{b} / N_{o}$ with $L=32,64,128, K=15$, and the processing window length of minimum reception interval. The extended $\mathrm{m}$-sequences defined in $[11]$ are used as the signature sequences. The LDMCDF with the negligible decision delay obviously outperforms the MD-MRC whose decision delay approaches infinity because of the performance gain provided by the decision-feedback detection. For example, to achieve the BER of $10^{-2}$ with $M=4$ resolvable paths, the MD-MRC requires about $3.5[\mathrm{~dB}]$ higher $E_{b} / N_{o}$ than the LDMCDF. In the case of the MD-MRC, the larger order of multipath diversity results in a higher decorrelating loss, which was previously observed in [10]. However, the LDMCDF preserves the larger diversity gain for the larger order of multipath diversity, since it applies the partial decorrelating operation to the combined multipath signals.

\section{CONCLUSIONS}

The multiuser detection for the asynchronous VPG-DS/CDMA system in mobile radio channels has been investigated without any restrictions on the processing gain. The equivalent synchronous single rate DS/CDMA system model has been derived. Based on the equivalent system model, the LDMCDF has been proposed. We have evaluated the effect of the processing window length on the performance of the LDMCDF. The simulation results show that the LDMCDF achieves good performance with the negligible decision delay at the cost of accurate estimation of the channel parameters. It is expected that the LDMCDF can be easily adapted to the changes in the various communication system parameters, such as the number of active users or channel parameters, since the proposed detection scheme is based on the equivalent synchronous single rate DS/CDMA system.

\section{REFERENCES}

[1] S. Moshavi, "Multi-user detection for DS-CDMA communications," IEEE Commun. Magazine, vol. 34, no. 10, pp. 124-136, Oct. 1996.

[2] T. Ottosson and A. Svensson, "Multi-rate schemes in DS/CDMA systems," in Proc. IEEE Vehic. Technol. Conf., Chicago, Illinois, USA, July 1995, pp. 1006-1010.

[3] M. Saquib, R. Yates, and N. Mandayam, "Decorrelating detectors for a dual rate synchronous DS/CDMA system," in Proc. IEEE Vehic. Technol. Conf., Atlanta, Georgia, USA, Apr. 1996, pp. 377-381.

[4] M. Saquib and R. Yates "A two stage decorrelator for a dual rate synchronous DS/CDMA system," in Proc. IEEE Int. Conf. Commun., Montréal, Québec, Canada, June 1997, pp. 334-338.

[5] M. Saquib, R. Yates, and N. Mandayam, "A decision feedback decorrelator for a dual rate synchronous DS/CDMA system," in Proc. IEEE Global Telecommun. Conf., London, UK, Nov. 1996, pp. 1804-1808.

[6] M. J. Juntti and J. O. Lilleberg, "Linear FIR multiuser detection for multiple data rate CDMA systems," in Proc. IEEE Vehic. Technol. Conf., Phoenix, USA, May 1997 pp. $455-459$.

[7] A. Duel-Hallen, "Decorrelating decision-feedback multiuser detector for synchronous code-division multipleaccess channel," IEEE Trans. Commun., vol. 41, no. 2, pp. 285-290, Feb. 1993.

[8] J. G. Proakis, Digital Communications, 3rd ed. Singapore: McGraw-Hill Book Co., 1995.

[9] G. H. Golub and C. F. Van Loan, Matrix Computations, 2nd ed. Baltimore: The Johns Hopkins University Press, 1991.

[10] Z. Zvonar and D. Brady, "Linear multipath-decorrelating receivers for CDMA frequency-selective fading channels," IEEE Trans. Commun., vol. 44, no. 6, pp. 650-653, June 1996:

[11] U. -C. G. Fiebig and M. Schnell, "Correlation properties of extended m-sequences," Electronics Letters, vol. 29, no. 20, pp. 1753-1755, Sept. 1993. 